\title{
PROPERTIES OF THE INTERSTELLAR MEDIUM AND THE PROPAGATION OF COSMIC RAYS IN THE GALAXY
}

\author{
A.D.Erlykin $\rrbracket^{2}$, A.A.Lagutin ${ }^{3}$, A.W.Wolfendale ${ }^{1}$ \\ (1) Department of Physics, University of Durham, South Road, Durham DH1 3LE, UK \\ (2) P. N. Lebedev Physical Institute, Leninsky Prosp., Moscow 117924, Russia \\ (3) Altai State University, Dimitrova st. 66, Barnaul 656099, Russia
}

\begin{abstract}
The problem of the origin of cosmic rays in the shocks produced by supernova explosions at energies below the so-called 'knee' ( at $\sim 3 \cdot 10^{6} \mathrm{GeV}$ ) in the energy spectrum is addressed, with special attention to the propagation of the particles through the inhomogeneous interstellar medium and the need to explain recent anisotropy results, [1]. It is shown that the fractal character of the matter density and magnetic field distribution leads to the likelihood of a substantial increase of spatial fluctuations in the cosmic ray energy spectra. While the spatial distribution of cosmic rays in the vicinity of their sources ( eg. inside the Galactic disk ) does not depend much on the character of propagation and is largely determined by the distribution of their sources, the distribution at large distances from the Galactic disk depends strongly on the character of the propagation. In particular, the fractal character of the ISM leads to what is known as 'anomalous diffusion' and such diffusion helps us to understand the formation of the Cosmic Ray Halo. Anomalous diffusion allows an explanation of the recent important result from the Chacaltaya extensive air shower experiment [1], viz. a Galactic Plane Enhancement of cosmic ray intensity in the Outer Galaxy, which is otherwise absent for the case of the so-called 'normal' diffusion. All these effects are for just one reason: anomalous diffusion emphasizes the role of local phenomena in the formation of cosmic ray characteristics in our Galaxy and elsewhere.
\end{abstract}

\section{Introduction Since the early fifties supernova remnants ( SNR ) have been} considered as the most likely sources of Galactic cosmic rays ( following the pioneering work of I.S.Shklovsky ). The main arguments for his proposal were sufficient energy deposition and the presence of non-thermal radio emission which indicates that SNR are the sites of electron acceleration. Later theoretical analysis of diffusive shock acceleration,

\footnotetext{
${ }^{1}$ Corresponding author: Erlykin A.D., e-mail: A.D.Erlykin@durham.ac.uk
} 
which was supposed to be the main mechanism of particle acceleration in SNR, confirmed that they can produce a power law spectrum of cosmic rays over a wide range of energies, and thus explain one of the most prominent features of the cosmic radiation.

Although there is evidence at $\mathrm{GeV}$ gamma ray energies for SNR acceleration of the initiating particles [2, 3], since the development of high energy ( $\mathrm{TeV}$ ) gamma ray astronomy doubts have begun to arise as to the role of SNR as the main sources of cosmic rays above some 10's of GeV. These doubts are inspired by the continuing absence of direct observations of $\mathrm{TeV}$ gamma-rays from known SNR which could be associated with the accelerated protons and nuclei. Another serious problem for the SNR model, and, indeed, for any model in which the source distribution is similar to that of SN, in terms of dependence of number on Galactocentric distance, is that the radial gradient of the proton and nuclei intensity in our Galaxy has been found to be less than the gradient of supernovae. Interestingly, the gradient for electrons - as determined from low energy gamma rays [4], and synchrotron radiation [5] - seems 'normal'.

Further problems are that the energy of the knee $\left(\sim 10^{6} \mathrm{GeV}\right)$ is above the maximum energy of accelerated protons in all models. Also, the required $\geq 10 \%$ efficiency of the energy transfer from the kinetic energy of the SNR shock to the energy of accelerated particles may well be too high in that the efficiency observed hitherto appears to be less than about $1 \%$ [6]. However, it must be said that the problem of maximum energy is less severe if, as we maintain, the knee is associated with 'heavy nuclei', specifically oxygen and iron [7, 8]. A way forward is to search for other sources of $\mathrm{TeV}$ cosmic ray nuclei ( eg [6] ) or to re-examine the manner in which cosmic rays propagate through the Galaxy, viz, whether or not the propagation has the usually-adopted Gaussian form ( spatially, about the source ). It is the latter approach that we adopt here. Specifically, we wish to see to what extent, the adoption of a more realistic model of cosmic ray diffusion in the ISM helps in the quest for an explanation of the following 'cosmic ray anomalies': the small cosmic ray gradient ( for protons and nuclei ), the extended cosmic ray halo, the Galactic Plane Enhancement in the Outer Galaxy and the energetics problem. However, indirectly we also test the basic conception that the main source of cosmic rays are SNR with their diffusive shock acceleration mechanism producing the energy spectrum with a power law exponent about 2.15 . 


\section{The properties of the interstellar medium ( ISM ) 2.1. Connection of the diffusion characteristics with the spectrum of magnetic turbulence in the ISM}

It is well known that the distribution of matter and magnetic fields in the Galaxy is highly non-homogeneous on different spatial scales. Gaseous clouds with very different densities, temperatures and degrees of ionization move through space in a highly turbulent way. Many evidences both from theory and observations of the existence of multiscale structures in the Galaxy have been found during a few last decades ( see, for instance, [9, 10, 11, 12, 13, 14, 15, 16, 17, 18, 19, 20, 21, 22, 23. Filaments, shells, clouds and other entities are widely spread in the ISM. A rich variety of structures which are created in interacting phases and have different properties can be related to the fundamental property of the turbulence, called the intermittency.

In 1941 A.V.Kolmogorov, assuming that all the characteristics of the turbulent medium depend only on the energy flux through the turbulent vortex hierarchy, derived an expression for the spectrum of turbulence ( the energy per unit mass and wave number bin, $k$ ). For steady-state hydrodynamic conditions, when the medium is incompressable, magnetic fields are absent and the energy flux does not depend on the viscosity, the spectrum is $F(k) \propto k^{-\chi}$ with $\chi=\frac{5}{3}$ [24]. At this point it is commonly assumed that not only is the Kolmogorov expression valid for cosmic ray propagation, but, furthermore, that the diffusion process is 'normal', viz. Gaussian. Specifically, for one-dimensional propagation we can write, for the particle density, $\rho$, at distance $x$ from a source and at time $t$ after a burst of particles is emitted:

$$
\rho(x, t)=\frac{1}{\sqrt{2 \pi \sigma}} \exp \left(-\frac{x^{2}}{2 \sigma^{2}}\right)
$$

where $\sigma=\sqrt{2 D t}, D$ being the diffusion coefficient. It will be shown that neither assumption is valid. Concerning the value of $\chi$, this changes if magnetic fields are present.

In the case of the gas being conductive, which is the usual state of the interstellar plasma, the turbulent motion generates magnetic fields and they, themselves, have a turbulent structure. The stretching, bending and folding of magnetic field lines by turbulent motions of the medium partially coupled to the field make the magnetic field also highly intermittent, especially on the smaller scales. 
Because magnetic irregularities scatter the charged particles, making the propagation of cosmic rays diffusion-like, the spectrum of these irregularities is important for the magnitude of the diffusion coefficient, which governs the transport of the cosmic rays. In 1965 R.H.Kraichnan showed that for the hydromagnetic case with the presence of magnetic fields the turbulence spectrum displays an exact equipartition between the magnetic and kinetic energies and its shape is modified. The predicted magnetic and kinetic energy spectra are both proportional to $k^{-\chi}$ with $\chi=\frac{3}{2}$ instead of $\frac{5}{3}$ [25].

As remarked already, many cosmic ray workers have used the Kolmogorov form - which has become a sort of 'standard' for cosmic ray diffusion; however, it is now appreciated that, insofar as most of the ISM is ionized to a significant degree, the Kraichnan form is more appropriate. In fact, we ourselves have prefered the latter 26] for an empirical reason, viz. that, when married with a 'standard' SNR production spectrum, the ensuing ambient cosmic ray spectrum has the 'correct' form.

The Galactic magnetic field is the superposition of a regular spiral component $B_{0}$ and a random component $B_{1}$, which is determined by turbulent motions in the ISM. The mean free path $\lambda_{s c}$ for the scattering of a particle with gyroradius $r_{g}$ by random magnetic irregularities with wave number $k$ is determined [27, 28] by

$$
\lambda_{s c}=\frac{r_{g} B_{0}^{2}}{B_{1}^{2}(k) k}
$$

Here $B_{1}^{2}(k)$ is the magnetic energy density contained in irregularities with wave number $k$ and, since it is determined by the ISM turbulence, $B_{1}^{2}(k) \propto k^{-\chi}$. Scattering of the particles is most efficient in the 'resonant' condition when their gyroradii, determined mostly by the regular magnetic field $B_{0}$, is close to the size of the irregularities, i.e. $r_{g} \approx 1 / k$. In this case the transverse diffusion across the regular magnetic field, which is important for the radial propagation of cosmic rays in the Galaxy, is characterized by the diffusion coefficient $D_{\perp}$, where $D_{\perp}$ depends on the energy $E$ as

$$
D_{\perp}(E)=D_{0} E^{\delta}
$$

$E$ nearly coincides with the rigidity for relativistic protons and electrons and

$$
\delta=2-\chi
$$


The parallel diffusion along the magnetic field lines which is typical for the cosmic ray propagation orthogonal to the Galactic disk ( there being evidence for field lines perpendicular to the Galactic Plane in the Halo ), is usually faster and goes with the parallel diffusion coefficient $D_{\|} \geq D_{\perp}[29$.

It is important to point out that the simple form for the energy dependence of the diffusion coefficient ( equation (3) ) can only apply below a particular energy, $E_{0}$, usually identified with that of the knee in the spectrum ( at $\sim 3 \mathrm{PeV}$ ). At higher energies where the spectrum steepens, it is usually considered that there is insufficient power in the irregularities to maintain the original value of $\delta$. The Kolmogorov case corresponds to the assumption of energy injection ( from SNR, etc. ) at the appropriate distance scale for $E_{0}$, viz. a few $p c$. At higher energies and bigger distance scales not only is the form of $D(E)$ very uncertain but near-straight line propagation is not far off.

\subsection{Anomalous diffusion in the fractal ISM}

Most calculations for the propagation of cosmic rays in the Galaxy have been made using 'quasilinear theory' [30], whose validity is limited to very low levels of turbulence and a quasi-homogeneous ISM. The turbulence and diffusion characteristics were connected only via the energy dependence of the diffusion coefficient determined by equations (3) and (4). In fact, as we mentioned in $\S 2.1$, the ISM has the multiphase character and is highly non-homogeneous. The turbulence level is quite high and the mean amplitude of the irregular magnetic field in the Galaxy determined by the turbulence is generally of the same order of magnitude as that of the regular field: $\frac{B_{1}}{B_{0}} \approx 1.5$ [31 and this ratio undoubtedly varies from place to place, depending on the proximity of stars of various types and 'shocks' of a wide range of strengths. The way forward - to advance on the simple homogeneous diffusion approximation - is frought with difficulty but we consider that one, hopefully fruitful, approach is by way of the so-called 'anomalous diffusion' scenario [32, 33, 34, 35]. Here there are two regimes: that of sub-diffusion, in which there are relatively small spatial displacements and of superdiffusion, where there are large displacements ( the so called 'Levy flights').

An approach to anomalous diffusive propagation has been recently developed by Lagutin et al. [36, 37]. In this work the power law character of the turbulence spectrum provides a self-similarity of the ISM structure, i.e. the ISM is fractal-like [38]. This 
self-similarity is not global and does not cover the whole Galaxy, but is valid on a wide range of spatial scales, at least in the pc and sub-pc scales of importance here. Indeed the experimental study of the ISM by radio observations revealed that various structures of the ISM are not distributed at random, but obey power-law relations between size, linewidth and mass [39]. These power laws demonstrate the self-similar nature of the ISM and are observed at the scales ranging from $100 \mathrm{pc}$ down to $2 \cdot 10^{-3} \mathrm{pc}$, i.e. at nearly 5 decades of the wave number $k$ from $2 \cdot 10^{-20}$ to $10^{-15} \mathrm{~cm}^{-1}$. It can be that the range is even wider, because direct measurements within the solar system made by Mariner-4 spacecraft found that above $k \approx 6 \cdot 10^{-12} \mathrm{~cm}^{-1}$ the wave number spectrum of the magnetic irregularities is proportional to $k^{-\frac{3}{2}}$ for another two decades [27].

On the basis of the assumption that the ISM has a fractal structure Lagutin et al. have formulated and solved analytically the equation for anomalous diffusion in a fractal medium for different input conditions. The basic equation for cosmic ray concentration $n$ without energy losses and nuclear interactions has the form

$$
\frac{d n}{d t}=-D(E, \alpha)(-\Delta)^{\frac{\alpha}{2}} n(r, t, E)+S(r, t, E)
$$

where $D(E, \alpha)$ is the anomalous diffusion coefficient, $\alpha$ is an exponent determined by the fractal structure of the medium, $(-\Delta)^{\frac{\alpha}{2}}$ is the fractional laplacian ( called 'Riss' operator [40] ). We remind that the normal diffusion is described by the similar equation, where the first term in its right part is $D(E) \Delta n(r, t, E), \Delta$ is an ordinary laplacian and the diffusion coefficient $D$ depends only on the energy. The details of the solution of the equation (5) are given in [36, 37].

The solution depends on the anomalous diffusion coefficient $D(E, \alpha)$ and the index $\alpha$ determines the temporal dependence of the cosmic ray propagation and the shape of the diffusion front. Later we show that its magnitude can be connected with the spectrum of magnetic irregularities. Therefore this index is the fundamental parameter for the propagation of cosmic rays in the turbulent ISM, which determines and interconnects the energy dependence and the speed of propagation as well as the shape of the diffusion front.

The standard diffusion in a homogeneous medium which leads to a Gaussian distribution of particle densities corresponds to $\alpha=2$. The case of $\alpha<2$ corresponds to the so-called superdiffusive regime of anomalous diffusion. 
It is appreciated that there is a certain ad hoc element in the treatment but, at least, the actual ingredients of the real ISM seem to be in place.

\section{The model}

Our model for particle acceleration by a SNR shock and the subsequent propagation through the ISM is actually a generalization of that described in [26]. The supernova explosions are assumed to be randomly distributed in time. Their spatial distribution in the Galactic disk is assumed cylindrically symmetric, as

$$
\rho_{S N}=A f(R, Z)
$$

where

$$
f(R, Z)=\left(\frac{R}{R_{\odot}}\right)^{a} \exp \left(-b\left(\frac{R}{R_{\odot}}-1\right)-\frac{Z}{H_{z}}\right)
$$

with $a=1.69 \pm 0.22, b=3.33 \pm 0.37, R_{\odot}=8.5 k p c, H_{z}=0.2 k p c$ 41. The normalization

constant $A$ has been taken to provide the total rate of $10^{-2}$ year $^{-1}$ Type II SN explosions in the Galaxy.

The acceleration and the propagation of cosmic rays during the SN explosion consists of two phases: the expansion and the diffusion. Details of the original model are given in [26]. In the present model the expansion phase of the explosion has not been changed and at the end of it the accelerated protons have a nearly power law energy spectrum with an exponent of about 2.2.

The diffusion phase of the propagation has been generalized to study the consequences of the different turbulence spectra. We have made calculations for 4 values of $\alpha: 2,1.5$, 1 and 0.5 . The important generalizations were related to the constraint:

$$
(D(E, \alpha) \tau)^{\frac{1}{\alpha}}=H_{G}
$$

and to the expression for the mean radius of the diffusive front:

$$
R_{d}=(D(E, \alpha) t)^{\frac{1}{\alpha}}=H_{G}\left(\frac{t}{\tau}\right)^{\frac{1}{\alpha}}
$$

Here $\tau$ is the mean lifetime of particles in the Galaxy which is determined by escape and $H_{G}$ is the vertical scale parameter of the Galaxy, which we adopt as $1 \mathrm{kpc}$ [26]. It is seen from (7) that the normal Gaussian case arises if $\alpha=2$ and that the temporal spread of the particles in the important cases of $\alpha<2$ is faster then for 'normal' diffusion. 
On the basis of a correspondence of Richardson's relative-diffusion law 42 : $R_{d} \propto t^{\frac{3}{2}}$ to the diffusion in the medium with Kolmogorov-type turbulence spectrum [43, 44] we assume that

$$
\alpha=2(2-\chi)=2 \delta
$$

and by this way connect the properties of the turbulent ISM with the characteristics of the cosmic ray propagation through it. The shape of the diffusion front is different for different $\alpha$ and is shown in Figure 1. It is seen that for $\alpha<2$ there is a tail at large distances due to particles which propagate with small scattering ( the 'Levy flights' referred to earlier ).

As for 'normal' diffusion the normalization of the particle density profile at any instant of time and in the absence of escape losses has been made to preserve the total energy contained in the cosmic rays equal to $10^{50} \mathrm{erg}$. The escape time has been determined by the constraint (8) and has a power law energy dependence with the same but negative exponent as $D(E, \alpha)$, i.e.

$$
\tau=\tau_{0} E^{-(2-\chi)}
$$

with $\tau_{0}=\frac{H_{G}^{\alpha}}{D_{0}}$.

\section{Energy spectra of cosmic rays}

The results for the energy spectra of cosmic rays calculated by us for different values of $\alpha$ are shown in Figure 2. 50 identical samples of 50000 SNR were simulated for 4 values of $\alpha: 2,1.5,1$ and 0.5. Better agreement between the observed and simulated spectra can be achieved by correcting the observed spectrum for the solar modulation effect at energies below $10 \mathrm{GeV}$. A higher absolute intensity in the simulations could be easily obtained using the higher SNR rate in the nearby Gould Belt and a higher efficiency for the energy transfer from kinetic energy of the explosion into accelerated cosmic rays [26]. It is seen that the mean spectra have different slopes, a result that arises because we connected the energy dependence of the escape time $\tau$ and the diffusion coefficient $D$ with the turbulence structure of the magnetic field in the ISM. The more uniform is the size distribution of magnetic irregularities the weaker is that dependence and the closer is the spectrum to that emerging after the acceleration at the end of the SNR expansion phase. 
We remark that the closest to the observed spectrum of cosmic rays at earth is that with $\alpha=1.0$ which corresponds to the turbulence spectrum derived by Kraichnan 25], a fact already referred to ( see $\S 2.1$ ). Also, among 50 different samples there is a good fraction which have a shape close to that observed below the knee, with its smoothly steepening background at $\mathrm{TeV}$ energies followed by a concave structure at higher energies. Such a structure of the spectrum has been explained by us in the Single Source Model [7, 8], where the 'knee' has structure due to a significant contribution from a local SNR. This situation is still true in the case of anomalous diffusion.

\section{Spatial distribution of cosmic rays}

\subsection{General remarks}

For the cosmic ray intensity at large distances above and below the Galactic Plane the stochastic character of the spatial distribution of SNR plays an insignificant role, because all SNR are concentrated in the disk itself. Thus, in our calculations of the intensity we have simply made a numerical integration over the smooth SNR distribution. The propagation of cosmic rays was calculated assuming that the turbulent properties of the ISM do not depend on the vertical ( $Z$ ) or radial $(R)$ distance and can be described by the same power law as within the Galaxy. In general this can be justified if, for instance, the regular and the mean irregular magnetic fields decrease with distance in such a way that the scattering mean free path (1) does not change.

\subsection{The radial distribution}

\subsubsection{General remarks}

In Figure 3a we show the distribution of cosmic rays out to large radial distances in the Galactic disk for two energies: $1 \mathrm{GeV}$ and $1 \mathrm{TeV}$. For comparison we show also the adopted distributions of SNR density normalized to 1 at its maximum. It is seen that the radial distribution of cosmic rays follows the radial distribution of SNR at almost all distances and for all values of $\alpha$, a fact due to the high sensitivity of cosmic ray intensity to nearby sources. The only exception is the region near the center of the Galaxy $(R \leq 2$ $\mathrm{kpc}$ ), where there is a lack of sources and cosmic rays fill in the gap. The significance of the result will be considered later.

\subsubsection{The proton radial gradient}

The gradient can be defined as $S=\frac{d(\ln I)}{d R}$, where I is the intensity of cosmic rays ( or 
the density of sources ). In Figure $3 \mathrm{a}$ we see that the radial gradient of cosmic rays is equal to the gradient of the sources, whatever the value of $\alpha$. At the site of the solar system, i.e. at $\mathrm{R}=8.5 \mathrm{kpc}$ it is $-0.17 \pm 0.05 \mathrm{kpc}^{-1}$. Experimental values for the Outer Galaxy derived by us from the gamma-ray emissivity profile are $(-0.10 \pm 0.02) \mathrm{kpc}^{-1}$ in Quadrant 2 and $(0.0 \pm 0.03) \mathrm{kpc}^{-1}$ in Quadrant 3 [46], ie the observed radial gradient is smaller than that predicted, a well-known fact [47. However as remarked in [47] the overall emissivity gradient does not necessarily hold locally. In particular, measurements of the emissivity in the Perseus arm at $3 \mathrm{kpc}$ from the Sun in the Outer Galaxy and at the distance of Cepheus and Polaris flare at $0.25 \mathrm{kpc}$ give a higher gradient: $-0.15 \pm 0.03$ $\mathrm{kpc}^{-1}$, which does not contradict the gradient of the SNR distribution [48. It is at greater galactocentric distances ( $R \geq 15 \mathrm{kpc}$ ) that the observed gradient is inconsistent with that predicted.

The maximum of the gamma-ray emissivity is at about the same distance, $R \approx 4$ kpc, from the Galactic center as the maximum of the SNR distribution which favors an SNR-origin of the bulk of the cosmic rays, or at least sources which have the same spatial distribution.

Since the amplitude of the anisotropy is $A=\lambda_{s c} S$ and we estimate from our model that for $1 \mathrm{TeV} \lambda_{s c} \approx 7.5 \cdot 10^{-3} \mathrm{kpc}$, then we expect that the anisotropy amplitude at this energy will be about $(1.3 \pm 0.4) \cdot 10^{-3}$, the experimental value being close to it: $(0.8 \pm 0.2) \cdot 10^{-3}$

To this extent, we can conclude that the anomalous ( superdiffusive ) diffusion mechanism does not help in elucidating the problem of the low overall radial gradient of protons and nuclei ( at least in the far Outer Galaxy ) and their association with SNR without additional assumptions ( we prefer re-entrant particles from the Halo - see 50]

\subsubsection{The electron radial gradient}

In contrast to the case for protons and nuclei there is no problem with th origin of cosmic ray electrons in SNR at least at energies below about some 10 's of GeV. The radial gradient at the site of the solar system ( derived from low energy gamma-rays ) is $-0.25 \pm 0.02 \mathrm{kpc}^{-1}$, which overlaps with the SNR gradient within the error bars 51 and with that predicted for 'cosmic rays' in general. The direct radio, X-ray and gamma-ray observations of SNR confirm the presence of accelerated electrons in the SNR shells. The 
estimated efficiency of the acceleration is about $1 \%$ which is much less stringent than the $\sim 10 \%$ required for protons. The reason for the difference between electrons and protons is not clear but it is perhaps due to a different radial dependence of the injection efficiency for the two particles.

\subsection{The vertical distribution}

Another situation is for the vertical direction. The Galactic disk is rather thin and the SNR distribution in this direction is narrow. The shape of the diffusion front is much more important in this case and Figure 3b shows that the longer tails of anomalous diffusion fronts create a much wider distribution, forming a cosmic ray halo, which spreads up to some tens of kpc outside the Galactic disk.

Interestingly, there is some evidence that there is significant ionized gas at large values of $Z$, specifically the mean gas density $\langle\rho\rangle \sim 10^{-3} \mathrm{~cm}^{-3}$ at $Z \sim 50 \mathrm{kpc}$ [52] and it has been suggested that there is a corresponding significant cosmic ray intensity [53]. Its value is not inconsistent with the value ( $\simeq 2 \cdot 10^{-5}$ of the Galactic Plane intensity ) found for $\alpha=1$ at $Z \sim 50 \mathrm{kpc}$. In fact such estimates are very approximate insofar as the diffusion coefficient $D_{\|}$is presumably greater in the outer halo than in the disk.

Further relevance of the $Z$-distribution is considered later.

\section{Galactic Plane Enhancement}

There is another effect connected with cosmic ray propagation - the possibility of observing the Galactic Plane Enhancement ( GPE ) in the Outer Galaxy. This quantity, $f_{e}$, was introduced by Wdowczyk and Wolfendale [54 to evaluate the deviation from cosmic ray isotropy in terms of its Galactic latitude distribution. We define this quantity in a somewhat different way from that in [54], by

$$
\frac{F(b)}{F_{0}}=\frac{\operatorname{Dgrad} I(b)}{c F_{0}}=\frac{2 H_{G}^{\alpha}\left(I(b)-I_{0}\right)}{c \tau \Delta^{\alpha-1} I_{0}}=a+f_{e} \cos ^{2} b
$$

Here $a$ is the fitting coefficient which should be equal to 1 for complete isotropy. $F(b)$ is the flux of cosmic rays from Galactic latitude $b$ and $F_{0}$ is the flux expected for an isotropic distribution. We have calculated these fluxes from the intensities $I_{0}$ at the solar system and $I(b)$ - at a distance of $\Delta=0.1 \mathrm{kpc}$ from the Sun in the direction of latitude $b$ and longitude $l=0^{\circ}$. In Figure 4 the ratio of these intensities is shown for an energy of $10^{5}$ $\mathrm{GeV}$. It is seen that for ordinary diffusion $(\alpha=2)$ the GPE is observed only in the 
Inner Galaxy $\left(b=0^{\circ}\right)$ and there is no sign of an enhancement in the Outer Galaxy $\left(b=180^{\circ}\right)$.

Decreasing $\alpha$, however, results in an increase of the GPE in terms of intensities both in the Inner and in the Outer Galaxy. The origin of this effect is in the much flatter radial distribution of sources compared with the vertical one. Due to the high sensitivity of the cosmic rays to the location of nearby sources, intensities at $b=0^{\circ}$ and $180^{\circ}$ are determined only by the radial gradient of SNR $S_{r} \approx-0.19 \mathrm{kpc}^{-1}$ for all values of $\alpha$ ( see Fig.3a ). The vertical gradient of SNR is much larger $S_{v} \approx-5 \mathrm{kpc}^{-1}$, however, and the intensity at $b=90^{\circ}$ is sensitive to the gradient of cosmic ray intensity $S_{v}^{\alpha}$ nearby the disk which is different for different $\alpha$. For instance, $S_{v}^{2}=-0.59 \mathrm{kpc}^{-1}, S_{v}^{1.5}=-0.81$ $\mathrm{kpc}^{-1}, S_{v}^{1}=-1.15 \mathrm{kpc}^{-1}$ and $S_{v}^{0.5}=-0.91 \mathrm{kpc}^{-1}$. Because the gradient is larger for smaller $\alpha$, the drop of intensity at $0.1 \mathrm{kpc}$ off the disk is higher and this overcomes the drop of intensity at the same $0.1 \mathrm{kpc}$ towards the Outer Galaxy at $b=180^{\circ}$. When one approaches the direction of $b=180^{\circ}$ from $90^{\circ}$ the intensity starts to rise again and the GPE becomes visible.

The experimental data on the anisotropy of EAS of the energy above $10^{5} \mathrm{GeV}$ observed at Chacaltaya shows clear evidence for the GPE in the Outer Galaxy [1]. Experimental points fitted by expression $(12)$ give $f_{e}=(1.18 \pm 0.48) \cdot 10^{-3}$. The same fitting of the curves shown in Figure 4 ( for the Outer Galaxy the latitude $b$ in the expression (12) is replaced by $b-\pi$ in radians ) give $f_{e}=(2.45 \pm 0.01) \cdot 10^{-5}$ for $\alpha=0.5,(1.02 \pm 0.01) \cdot 10^{-3}$ for $\alpha=1$ and $(1.28 \pm 0.03) \cdot 10^{-2}$ for $\alpha=1.5$. As seen in Figure 4 there is no GPE for $\alpha=2$ and the formally determined $f_{e}$ has a negative value.

When comparing the experimental and theoreticasl values of GPE one has to keep in mind the fact that the experimental value has been derived from the fluxes averaged over wide intervals of Galactic longitude. However, it should not have a considerable effect: in the experiment [1] the averaging has been made over a $90^{\circ}$ longitude band centered on $l=180^{\circ}$. Our calculations show that the value $I(l, b)-I_{0} \propto \cos \left(l-180^{\circ}\right)$ so that the averaging over the interval of $l=135^{\circ}-225^{\circ}$ can diminish our $f_{e}$ by no more than $10 \%$.

The comparison shows that the closest approach to the experimental value of $f_{e}$ is again achieved for $\alpha=1.0$. However, stochastic variations of the cosmic ray intensity due to the proximity of nearby sources can spoil this agreement. 
The most important conclusion from this consideration and Figure 4 is that the propagation in the non-homogeneous medium gives an explanation of the observed positive value of Galactic Plane Enhancement in the Outer Galaxy, whereas the 'normal' diffusion mechanism fails here.

A general point that can be made here concerns the shape of $I(b)$. Our model gives a good fit to the experimental data and the empirical function (equation (12)). Detailed calculations have not yet been made but it seems likely that such agreement can be used to give an upper limit to the $Z$-distribution of the sources. Such arguments will be useful as a diagnostic for possible source types. At present we can rule out source distributions with $\langle Z\rangle>$ several kpc and thus 'objects' which have been projected out into the Halo and large scale acceleration in the Halo itself.

\section{Local deviations of the cosmic ray intensity}

In our previous work [26] we emphasized the point that there is a high sensitivity of the observed cosmic ray intensity and its spectral shape to the local spatial and age distribution of SNR and here we have shown that this sensitivity rises with decreasing $\alpha$. In Figure 5 we show examples of the Galactic latitude distributions for random distributions of SNR simulated within a radius of $0.5 \mathrm{kpc}$ from the solar system added to the regular distribution from the more distant SNR taken within $10 \mathrm{kpc}$ radius. There are 12 independent samples of 1000 random supernovae in each sample and they were simulated using 4 types of ISM with $\alpha=2,1.5,1$ and 0.5 .

It is seen again that the fluctuations increase for lower values of $\alpha$ and there are samples which exhibit a completely different latitude dependence with a 'negative enhancement' or a displaced phase of an enhancement. Among the latter there are some samples which demonstrate maxima at $l>180^{\circ}$. There is a south-north asymmetry observed in the experiment [1] and a small displacement of the peak intensity ( to $b \sim 200^{\circ}$ ) and our simulations indicate that these features could have a local origin.

\section{Conclusions}

We have shown that the structure of the ISM can determine some of the important characteristics of the cosmic rays in our Galaxy: the slope of the energy spectrum and its fluctuations, the formation of the halo at large distances from the Galactic disk, the presence of a Galactic Plane Enhancement and a south-north asymmetry in the Outer 
Galaxy. All our simulations have been made within the framework of the conception that SNR are the sources of cosmic rays in our Galaxy and diffusive shock acceleration is the mechanism of particle acceleration at energies below the knee. We emphasize the importance of the local structure of the ISM for the formation of most cosmic ray characteristics. A comparison of the simulated characteristics with experiment indicates that the fractal structure of ISM with the parameter $\alpha=1$ (Kraichnan spectrum of magnetic irregularities ) gives local cosmic ray characteristics which are closest to the experiment.

Returning to the specific questions referred to in $\S 1$, the answers are as follows: The radial gradient: the sole 'new model' does not help, the explanation needs additional assumptions.

Energetics: there is no improvement.

GPE in the Outer Galaxy: here there is an improvement. The observations are consistent with the anomalous diffusion model but not with 'normal diffusion'.

As a bonus we have the Halo and the greater ease of explaining the spectral shape.

\section{Acknowledgements}

The authors are grateful to the UK's Particle Physics and Astronomy Research Council, to The Royal Society and to The Russian Foundation for Basic Research for financial support and an unknown referee for the useful remarks.

\section{References}

[1] Y.Tsunesada et al., 2001, Proc. 27th Int. Conf. Cosm. Rays, Hamburg, 1, 10

[2] C.L.Bhat et al., 1985, Nature, 314, 511 and 515

[3] J.L.Osborne et al., 1995, J. Phys. G: Nucl. Part. Phys., 21, 429

[4] M.R.Issa, A.W.Wolfendale, 1981, J. Phys. G: Nucl. Part. Phys. 7, L187

[5] A.Broadbent, 1989, PhD Thesis, Univ. of Durham

[6] R.Plaga, 2001, astro-ph/0111555

[7] A.D.Erlykin, A.W.Wolfendale, 1997, J. Phys. G: Nucl. Part. Phys., 23, 979 
[8] A.D.Erlykin, A.W.Wolfendale, 2001, J. Phys. G: Nucl. Part. Phys., 27, 1005

[9] L.C.Lee, J.R.Jokipii, 1976, Astrophys.J., 206, 735

[10] S.A.Kaplan, S.B.Pikelner, 1979, Physics of the Interstellar Medium, M.,Nauka ( in Russian )

[11] T.A.Lozinskaya, 1986, Supernova and star wind: interactions with galactic gas, M.,Nauka ( in Russian )

[12] A.A.Ruzmaikin, Sokolov D.D., Shukurov A.M., 1988, Magnetic fields of galaxies, Kluwer, Dordrecht

[13] S.I.Vainstein, Bykov A.M., Toptygin I.N., 1989, Turbulence, stream layers and shock wave in the cosmic plasma, M.,Nauka (in Russian )

[14] N.G.Bochkarev, 1990, The local interstellar medium, M.,Nauka ( in Russian )

[15] E.Falgarone, T.G.Phillips, C.K.Walker, 1991, Astrophys. J., 378, 186

[16] L.F.Burlaga, J.Perko, J.Pirraglia, 1993, Astrophys. J., 407, 347

[17] S.A.Molchanov, A.A.Ruzmaikin, D.D.Sokolov, 1993, Nonlinear waves: physics and astrophysics, M.,Nauka ( in Russian )

[18] M.S.Meish, J.Bally, 1994, Astrophys. J., 429, 645

[19] J.W.Armstrong, B.J.Rickett, S.R.Spangler, 1995, Astrophys. J., 443, 209

[20] B.G.Elmegreen, E.Falgarone, 1996, Astrophys. J., 471, 816

[21] A.H.Minter, S.R.Spangler, 1996, Astrophys. J., 458, 194

[22] A.C.Cadavid, Lawrence J.K., Ruzmaikin A.A., 1999, Astrophys. J., 521, 844

[23] B.G.Elmegreen, Kim S., Staveley-Smith L., 2001, Astrophys. J., 548, 749

[24] A.V.Kolmogorov, 1941, C.R. Acad. Sci. USSR, 30, 301

[25] R.H.Kraichnan, 1965, Phys. Fluids, 8, 1385 
[26] A.D.Erlykin, A.W.Wolfendale, 2001, J. Phys. G: Nucl. Part. Phys., 27, 941

[27] M.S.Longair, 1992, High Energy Astrophysics, Second edition, v.1, Cambridge University Press

[28] V.S.Berezinsky et al. 1984, Astrophysics of Cosmic Rays, Moscow, Nauka

[29] F.Casse et al., 2001, Phys. Rev. D, 65, 023002

[30] R.Jokipii, 1966, Astrophys. J., 146,480; ibid. 1973, 183, 1029; ibid. 1987, 313, 842

[31] A.Broadbent et al., 1990, Proc. 21st Int. Cosm. Ray Conf., Adelaide, 3, 229

[32] J.P.Bouchaud, A.Georges, 1990, Phys. Rep., 195, 127

[33] M.B.Isichenko, 1992, Rev. Mod. Phys., 64, 961

[34] B.J.West, W.Deering, 1994, Phys. Rep., 246, 1

[35] V.V.Uchaikin, V.M.Zolotarev, 1999, Chance and stability, VSP, Netherlands, Utrecht

[36] A.A.Lagutin et al., 2001, Nucl. Phys. B ( Proc.Suppl. ), 97, 267

[37] A.A.Lagutin et al., 2001, Proc. 27th Int. Cosm. Ray Conf., Hamburg, 5, 1900

[38] Feder J., 1988, Fractals, Plenum Press, New York and London

[39] Combes F., 1999, Celestial Mechanics and Dynamical Astronomy, 72, 91

[40] Samko S.G., Kilbas A.A., Marichev O.I., 1987, Fractional integrals and derivations and some applications, Minsk, Nauka ( in Russian )

[41] M.Pohl, J.A.Esposito, 1998, Astrophys. J., 507, 327

[42] L.F.Richardson, 1926, Proc. Roy. Soc. London, Ser.A 110, 709

[43] G.K.Batchelor, 1953, The theory of homogenous turbulence, Cambridge Univ. Press.

[44] M.Lesieur, 1990, Turbulence in Fluids, Kluwer Acad. Publ.

[45] A.V.Apanasenko et al. 2001, Astropart. Phys., 16, 13 
[46] A.D.Erlykin et al., 1996, Astron. Astrophys. Suppl. Ser., 120, 397

[47] J.Paul, 2000, High Energy Gamma-Ray Astronomy, Int. Symp., Heidelberg, AIP Conf. Proc. 558, 183

[48] S.W.Digel et al., 1996, Astrophys. J., 463, 609

[49] A.A.Watson, 1984, Adv.Space Res., 4, 35

[50] A.D.Erlykin, A.W.Wolfendale, 2002, J. Phys. G: Nucl. Part. Phys., 28, 2329

[51] K.Beuerman et al., 1985, Astron. Astrophys.,153, 17

[52] B.Moore, M.Davis, 1994, Mon. Not. Roy. Astron. Soc., 207, 209

[53] J.Wdowczyk, A.W.Wolfendale, 1995, Proc. 24th Int. Cosm. Ray Conf., Rome, 3, 360

[54] J.Wdowczyk, A.W.Wolfendale, 1984, J. Phys. G: Nucl. Part. Phys., 10, 1453 


\section{Captions to figures}

Figure 1. Shape of the particle density distribution for different values of the $\alpha$ parameter. The curves are normalized at $x=0, x$ is the ratio of distance from the source to the mean distance. The long 'tail' for anomalous diffusion will be noted.

Figure 2. Energy spectra of cosmic rays for different values of the $\alpha$-parameter. 50 samples of 50000 SNR are simulated in each case. The region between the two thick full lines, denoted as 'obs', is the observed cosmic ray spectrum 45.

Figure 3. The radial (a) and vertical (b) lateral distribution of the cosmic ray intensity for energies $1 \mathrm{GeV}$ ( thick lines) and $1 \mathrm{TeV}$ ( thin lines ) for different values of the $\alpha$-parameter. Full line: $\alpha=2$, dashed line: $\alpha=1.5$, dash-dotted line: $\alpha=1$ and dash-3dotted line: $\alpha=0.5$. Dotted line - SNR distribution.

Figure 4. The Galactic latitude distribution of the cosmic ray intensity for the energy $10^{5} \mathrm{GeV}$ and different values of the $\alpha$-parameter. Full line: $\alpha=2$, dashed line: $\alpha=1.5$, dash-dotted line: $\alpha=1$ and dash-3dotted line: $\alpha=0.5$. The longitude $l$ has been taken as $0^{\circ}$ for the Inner Galaxy and $180^{\circ}$ for the Outer Galaxy. The GPE is an excess at $b=0^{\circ}$ and/or $180^{\circ}$.

Figure 5. The galactic latitude distribution of the relative cosmic ray intensity for energy $1 \mathrm{TeV}$ and different values of the $\alpha$-parameter: 2 (a), 1.5 (b), 1 (c) and 0.5 (d). Intensities include contributions from SNR randomly distributed in space and time within $0.5 \mathrm{kpc}$ from the Sun. 
This figure "pro1.gif" is available in "gif" format from: http://arxiv.org/ps/astro-ph/0209506v1 
This figure "pro2.gif" is available in "gif" format from: http://arxiv.org/ps/astro-ph/0209506v1 
This figure "pro3.gif" is available in "gif" format from: http://arxiv.org/ps/astro-ph/0209506v1 
This figure "pro4.gif" is available in "gif" format from: http://arxiv.org/ps/astro-ph/0209506v1 
This figure "pro5.gif" is available in "gif" format from: http://arxiv.org/ps/astro-ph/0209506v1 